\title{
Warum besucht man einen Homöopathen? Eine Praxis in Münster im 19. Jahrhundert
}

Marion Baschin

\section{Summary}

The physician Friedrich von Bönninghausen (1828-1910) practiced from 1864 until his death as a homoeopath in Münster/Westphalia. The article locates this "alternative" practice in its medical surrounding. It is shown which cures the patients had already used before they turned to the method of Hahnemann and which motives are given in the records for their decision to consult the homoeopath.

Keywords: Bönninghausen, homoeopathy, medical market, patient behavior

\section{Zusammenfassung}

Der Arzt Friedrich von Bönninghausen (1828-1910) führte von 1864 bis zu seinem Tod eine homöopathische Praxis in Münster/Westfalen. Diese «alternative» Praxis wird in ihr medizinisches Umfeld eingeordnet. Dabei wird aufgezeigt, welche Kuren die einzelnen Patienten genutzt hatten, ehe sie sich der Lehre Hahnemanns zuwandten, und welche Motive sich in den einzelnen Krankengeschichten für die Entscheidung, den Homöopathen zu konsultieren, erkennen lassen.

Schlagworte: Bönninghausen, Homöopathie, medizinischer Markt, Patientenverhalten

Dr. Marion Baschin, Institut für Geschichte der Medizin der Robert Bosch Stiftung, Straußweg 17, D-70184 Stuttgart (marion.baschin@igm-bosch.de). 
Der Arzt und Homöopath Friedrich von Bönninghausen praktizierte zwischen 1864 und 1910 in Münster, der Hauptstadt der preußischen Provinz Westfalen. ${ }^{1}$ Im Folgenden geht es zunächst darum, seine Praxis in das medizinische Umfeld der Stadt einzuordnen. Dann steht die Frage im Mittelpunkt, welche Kuren die Kranken in Anspruch genommen hatten, bevor sie zu dem «alternativen» Angebot griffen. Abschließend kommen einige Motive der Patienten, sich einer homöopathischen Kur zuzuwenden, zur Sprache. Dies trägt dazu bei, deren Verhalten im Rahmen des medizinischen Marktes zu erhellen.

\section{Münsters medizinischer Markt aus Sicht des Arztes}

Als Friedrich von Bönninghausen 1862 in Münster approbiert wurde, trat er in einen stark besetzten medizinischen Markt ein. Er war zu diesem Zeitpunkt einer von insgesamt 34 Ärzten in der Stadt Münster, die im Schnitt etwa 680 Einwohner betreuten. ${ }^{2}$ Friedrich hatte den Vorteil, dass er nach dem Tod seines Vaters 1864 dessen Praxis weiterführen konnte. So verfügte er bereits über einen gewissen Patientenstamm und hatte sich praktische Erfahrung aneignen können. ${ }^{3}$ Doch die Konkurrenz mit anderen «Kollegen» war groß. Die Spezialisierung Bönninghausens auf die homöopathische Therapie bot ihm einerseits eine Nische, andererseits bedeutete sie eine gewisse Einschränkung, da die Heilmethode zumindest eine interessierte oder eine teilweise überzeugte Klientel voraussetzt.

Prinzipiell standen den Bürgern in Münster und der Provinz Westfalen für die Krankenbehandlung verschiedene Möglichkeiten offen. Nach den ersten Gefühlen des Unwohlseins dürften zunächst fast alle «Kranken» auf die medizinische Selbstversorgung zurückgegriffen oder von Familie und Bekannten Rat geholt haben. ${ }^{4}$ Auch wurden häufig nicht zugelassene Laien-

1 Seine Tätigkeit ist aber nur bis 1889 durch handschriftlich geführte Krankenjournale belegt. Diese sind überliefert in IGM Bestand P 116-149. Zu deren Aufbau Baschin 2010, 39-41. Im Rahmen der Untersuchung wurden insgesamt 6832 Krankengeschichten aus diesen Journalen erfasst und ausgewertet.

2 Zur Ärztedichte in Münster bis 1864 ausführlicher Baschin 2010, 403. Damals hatte Münster 23124 Einwohner. Teuteberg 1993a, 23-24.

$3 \mathrm{Zu}$ den Startschwierigkeiten junger Ärzte in dieser Zeit Huerkamp 1985, 119-126. Der Vater war Clemens Maria Franz von Bönninghausen (1785-1864). Zu dessen Praxis Baschin 2010. Es ist nichts darüber bekannt, wie Friedrich von Bönninghausen weitere Patienten «anwarb». Siehe hierzu auch die folgenden Ausführungen im Zusammenhang mit den Informationen in Adressbüchern.

4 Die Frage, ab wann eine Person sich überhaupt «krank» fühlt, ist ausgesprochen schwer zu beantworten und sehr individuell. Baschin 2010 mit weiterer Literatur. 
heiler, sogenannte «Quacksalber» oder «Kurpfuscher», konsultiert. ${ }^{5}$ In Münster und der Umgebung boten diese im 19. Jahrhundert ebenfalls ihre Dienste an. Quantitative Aussagen über die Anzahl von Laienheilern und das Ausmaß von deren Praxen sind aber kaum möglich. ${ }^{6}$

Von Rechts wegen waren die approbierten Ärzte für die Behandlung Kranker zuständig. ${ }^{7}$ Im Jahr 1852 schuf die neue preußische Prüfungsordnung den ärztlichen «Einheitsstand». Entsprechend führte Friedrich von Bönninghausen, so wie fast alle seine «Konkurrenten», den Titel «praktischer Arzt, Wundarzt und Geburtshelfer». ${ }^{8}$ Im Gegensatz zu ihren Vorgängern in der Frühen Neuzeit definierten diese sich nicht mehr als «Gelehrte», die der Welt der «Gebildeten und dem damit verbundenen Lebensstil» zugehörten, sondern durch ihre Berufstätigkeit als «Praktiker», die in «allen Feldern der Heilkunde bewandert» waren und die Verantwortung für ihre Patienten übernahmen, indem sie auch unter widrigen Umständen den Betroffenen zu Hilfe eilten. ${ }^{9}$

Die Arztdichte war in Münster das gesamte 19. Jahrhundert hindurch hoch und lag über dem preußischen Landesdurchschnitt. ${ }^{10}$ Dies hängt nicht nur mit dem bekannten Stadt-Land-Gefälle in der medizinischen Versorgung zusammen. Münster war als Provinzialhauptstadt mit einem großen Anteil von dort wohnenden Geistlichen, Beamten und Adeligen im Hinblick auf die Verdienstmöglichkeiten und die potentielle Kundschaft besonders attraktiv. ${ }^{11}$ In den 1860er Jahren nahm die Anzahl der Einwohner je Arzt auch in Münster gegenüber dem Beginn des 19. Jahrhunderts leicht zu. Insbesondere im Zuge der Eingemeindung 1875 stieg die Bevölkerungszahl Münsters stark an, während die Anzahl der niedergelassenen Ärzte etwa gleich blieb. Daher hatte in den 1880er Jahren ein Arzt annähernd 1200 Einwohner zu betreuen. ${ }^{12}$ Dieses Verhältnis besserte sich erst in den

5 Zu den Begriffen Spree 1989.

6 Einen Überblick bieten Schwanitz 1990, 125-127, sowie Baschin 2010, 101-107.

7 Zur Beschreibung der medizinischen Situation im 19. Jahrhundert und der Rolle der «akademischen» Ärzte Huerkamp 1985, Lachmund/Stollberg 1995, 67-130, Loetz 1993 oder Jütte 1997.

8 Zu dem Prozess Huerkamp 1985, bes. 45-59. Zum Preußischen Medizinalwesen Eulenberg 1874.

9 Huerkamp 1985, 58. Zu dem Topos des «Landarztes» ferner Dinges 2008, 23f. mit weiterer Literatur.

10 Schwanitz 1990, 117.

11 Huerkamp 1985,51 und 138-141, Teuteberg 1993b, 362. Zur Sozialstruktur der Stadt Münster und ihrem Stadtcharakter Krabbe 1983.

12 Die «gravierende Unterversorgung» mit Ärzten für Preußen in den 1860er Jahren machte sich daher in Münster selbst zunächst nicht so stark bemerkbar. Für Preußen lag die Quote bei etwa 3000 Einwohnern je Arzt im Jahr 1863 und verschlechterte sich bis 1876 auf knapp 3400 Einwohner je Arzt. Huerkamp 1985, 110. 
1890er Jahren wieder, als sich in Münster, wie auch in Preußen generell, die Lage entspannte. ${ }^{13}$

Seit 1875 zeichneten sich die ersten Spezialisierungstendenzen in der Medizin ab. Neben Bönninghausen ließen sich ab 1878 weitere homöopathische Ärzte in Münster nieder und sorgten so für Konkurrenz im Feld dieser «Außenseitermethode». ${ }^{14}$ Einzelne Ärzte machten in den Adressbüchern der Stadt gezielt auf ihre besondere Qualifikation, wie die Ausübung der Augenheilkunde oder die Behandlung von Hals-Nasen-Ohren-Krankheiten, aufmerksam. ${ }^{15}$ Auch hinter dem Namen Friedrichs von Bönninghausen wies die Bezeichnung «Homöopath» in Klammern auf dessen «alternative» Behandlungsweise hin. Doch im Gegensatz zu anderen Ärzten veröffentlichte er keine Informationen über Sprechstunden und besaß später auch keinen Telefonanschluss. ${ }^{16}$

Die Stadt Münster verfügte bis 1864 über sechs Krankenhäuser und Einrichtungen, in denen Kranke versorgt wurden. ${ }^{17}$ In den folgenden Jahren wurden kleinere Spezialkliniken ins Leben gerufen, und die bestehenden Institutionen erweiterten ihre Aufnahmekapazitäten. ${ }^{18}$ Zudem gab es Hebammen, wobei deren Anzahl nur langsam zunahm, so dass zu Beginn des 20. Jahrhunderts mehr Einwohner von einer Hebamme versorgt werden mussten als zuvor. ${ }^{19}$ Zunächst gab es bis in die 1880er Jahre hinein fünf Apotheken. Deren Anzahl erhöhte sich bis 1900 auf sieben. ${ }^{20}$ Bönning-

13 So versorgten in Münster im Jahr 189568 Ärzte 57135 Einwohner (840 Einwohner je Arzt).

14 Es handelt sich um August Petrasch (1805-1893), Hermann Schnütgen (1846-1919), Bernhard Sanders (1863-1916) und Robert Schnütgen (1877-1963).

$15 \mathrm{Zu}$ der Spezialisierung der praktischen Ärzte Huerkamp 1985,177-185. Münster stellte damit keine Ausnahme dar. Vielmehr waren spezialärztliche Praxen ein Großstadtphänomen.

16 Vergleiche beispielsweise die Einträge im Adreß-Buch 1889, 38 (Schnütgen) oder AdreßBuch 1905, 89 (Sanders). Insofern muss die Frage, ob Bönninghausen aktiv etwas zur Gewinnung von Patienten unternahm, eher verneint werden. Weitere Informationen gibt es diesbezüglich aber nicht.

17 Schwanitz 1990.

18 Diese ergänzten die konfessionellen Krankenhäuser. Hierzu Jungnitz 1981, 180-182, Schwanitz 1990, 91-100. Die Entwicklung in Münster fügt sich damit nahtlos in die gesamtdeutsche Entwicklung. Spree 1996, 60-62.

19 Schwanitz 1990, 127f. In der Stadt Münster gab es 1865 beispielsweise 12 Hebammen, im gesamten Regierungsbezirk Münster waren 303 Hebammen tätig. StAM Regierung Münster 203 I Nachweise der Medicinal-Personen Band 1 1865, 292 (Stadt) und 295 (Regierungsbezirk). 1895 gab es in Münster 25 Hebammen, die im Durchschnitt jeweils 2900 Einwohner versorgten. StAM Regierung Münster 866 Generalbericht über das Medizinalwesen im Regierungsbezirk Münster 1874-1901, 202.

20 Im Jahr 1865 verfügte der gesamte Regierungsbezirk Münster über 62 Apotheken. StAM Regierung Münster 203 I Nachweise der Medicinal-Personen Band 1 1865, 295. StdAM Stadtregistratur Medizinalangelegenheiten Fach 202 Nr. 8 Nachweise der Medizinalpersonen, Band 5, 1895-1906, 204. 
hausen selbst durfte eine Hausapotheke führen, die im Rahmen der üblichen Visitationen durch die Behörden geprüft wurde. ${ }^{21}$

Der gesamte medizinische Markt unterlag ständiger Beobachtung und Gestaltungsversuchen von staatlicher Seite. ${ }^{22}$ Bönninghausen war in diese staatlichen Strukturen durch seine Approbation eingebunden, wobei er beispielsweise die 1881 eingeführten «Zählkarten über gemeingefährliche Krankheiten» ausfüllen musste. ${ }^{23}$ Die Forderung der Ärzte nach einer staatlich anerkannten Standesvertretung wurde in Preußen erst 1887 mit der Bildung der Ärztekammer erfüllt. ${ }^{24}$ Friedrich von Bönninghausen war zwar wahlberechtigt, machte davon aber wohl keinen Gebrauch, da der Umgangston zwischen der Westfälischen Ärztekammer und den bekennenden Homöopathen zunehmend rauer wurde..$^{25}$

Der «Ärztliche Verein der Stadt Münster» war 1872 gegründet worden. Auch auf der Ebene des Regierungsbezirks und der Provinz gab es entsprechende Organisationen. ${ }^{26}$ Friedrich von Bönninghausen war in keinem der Vereine Mitglied. Einerseits wäre er als Homöopath wohl nicht in einer solchen Organisation willkommen gewesen, andererseits scheint er ebenso wenig an einem «kollegialen» Austausch interessiert gewesen zu sein. ${ }^{27}$ Die homöopathisch praktizierenden Ärzte hatten sich ebenfalls in Vereinen zusammengeschlossen und versuchten so, ihre Interessen zu bündeln und den wechselseitigen Austausch zu fördern. Doch auch in diesen wirkte Bönninghausen nicht mit. ${ }^{28}$

Mit der Einführung der gesetzlichen Krankenversicherung ab 1884 wurden die Krankenkassen zu einem weiteren Faktor in der Arzt-Patien-

21 StAM 893 V-236: Die Homöopathie und die homöopathischen Ärzte, 1854-1895, 63. StdAM Medizinalangelegenheiten Fach 202 Nr. 3: Überprüfung der Apotheken und Drogerien wegen der Arzneitaxe, 1817-1924, 283.

22 Die entsprechende Behörde war das Provinzialmedizinalkollegium beziehungsweise die Abteilungen für das Medizinalwesen innerhalb der Regierung. Walter 1993, 55. Zu den rechtlichen Grundlagen und gesetzlichen Regelungen in Preußen Eulenberg 1874.

23 StdAM Stadtregistratur Fach 204 Nr. 11 Zählkarten über gemeingefährliche Krankheiten 1881.

24 In anderen Ländern wurden entsprechende Ärztekammern früher eingeführt. Huerkamp 1985, 261-264.

25 StdAM Stadtregistratur Medizinalangelegenheiten Fach 202 Nr. 7: Einrichtung der Ärztekammer, 1887-1922, 9. Bei der Sitzung der Ärztekammer am 21. Oktober 1902 hatte man die «consultative Behandlung mit Homöopathen» abgelehnt und jene als «Kurpfuscher» bezeichnet. Bönninghausen wird auf der Liste der Wahlberechtigten geführt. Eine Stimmabgabe wurde aber nicht verzeichnet.

26 Graf 1890, 146f.

27 Die Homöopathen Schnütgen und Sanders wurden zu Beginn des 20. Jahrhunderts nicht in den «ärztlichen Standesverein» aufgenommen, weil sie «nicht auf dem Boden der Naturwissenschaften» stehen würden. Sanders 1964, 339.

28 Das homöopathische Vereinswesen in Westfalen nach Stahl 1995. Der Zentralverein homöopathischer Ârzte war 1829 gegründet worden. Hierzu Schlich; Schüppel 1996, bes. 215-217. In keinem der überlieferten Mitgliederverzeichnisse wurde der Name Bönninghausens gefunden. 
ten-Beziehung. Friedrich von Bönninghausen hat aber nicht als Kassenarzt praktiziert, sondern Patienten privat behandelt. Auch von Kostenübernahmen durch die Armenkasse ist nichts bekannt. ${ }^{29}$

\section{Münsters medizinischer Markt aus Sicht der Patienten}

Homöopathen legen im Rahmen der ausführlichen Anamnese besonderen Wert auf Informationen über bereits eingenommene Medikamente oder durchgeführte Behandlungen. Dies ist der Tatsache geschuldet, das hierdurch die ursprünglichen Symptome, welche für die Wahl des passenden Arzneimittels entscheidend sind, verändert worden sein könnten. ${ }^{30}$ Friedrich von Bönninghausen beherzigte diese Hinweise ebenfalls und vermerkte in den Krankenjournalen eine vorangegangene Mitteleinnahme, eine frühere therapeutische Maßnahme oder einen vorherigen Kurversuch. Aus diesen lässt sich der medizinische Markt aus Sicht der Patienten zumindest teilweise rekonstruieren. ${ }^{31}$

Prinzipiell hatte etwas mehr als die Hälfte aller Patienten von Bönninghausen vor der homöopathischen Behandlung eine andere Therapie versucht. ${ }^{32}$ Die überwiegende Mehrheit dieser Betroffenen hatte Medikamente eingenommen, wobei nicht klar ist, inwieweit es sich um eine Selbstmedikation oder die Verordnung durch einen Arzt handelte. ${ }^{33}$ Die Mittel der Selbstmedikation unterschieden sich oft nicht von denen der «Schulmedizin». ${ }^{34}$ Das Spektrum reichte von den traditionellen, bewährten Heilmitteln und Bädern bis zu der Einnahme von Tabletten und Mixturen,

29 Für Friedrich von Bönninghausen konnte keine Position in Staatsdiensten, hierzu würde auch die Stelle als Armenarzt zählen, oder als Kassenarzt nachgewiesen werden. Die Rechnungsbeträge und Vermerke über deren Bezahlung in IGM Conto-Buch P 156. In keiner der berücksichtigten Behandlungen findet sich ein Hinweis auf Krankenkassen.

30 Hahnemann 1999, 171 (§ 86). Auch andere Ärzte waren an der Vorbehandlung ihrer Patienten interessiert, beispielsweise Balster 1990, 202-208.

31 Friedrich von Bönninghausen notierte diese Angaben entweder in die eigens dafür vorgesehene Zeile unter «Allop.(athisch) gebr.(aucht)» oder im Zusammenhang mit den Krankheitsmerkmalen.

32 Bei 2977 (43,6\%) der 6832 Betroffenen war keine entsprechende Angabe gemacht worden. 232 der Befragten $(3,4 \%)$ hatten keinen vorherigen Therapieversuch unternommen. Bei 3623 Patienten $(53,0 \%)$ waren diesbezügliche Informationen vorhanden.

33 Von 2952 Patienten wurden auf die Frage Bönninghausens nach den vorangegangenen Kuren ein oder mehrere Medikamente genannt. In den allermeisten Fällen gibt es aber keine näheren Angaben dazu. Dies sind 43,2\% aller Kranken und 81,5\% von denjenigen Patienten, die vorher «allopathisch» behandelt wurden. Bei 212 der Betroffenen ist ein Arzt im Zusammenhang mit der vorangegangenen Kur erwähnt. Die Vermutung liegt nahe, dass in diesen Fällen eine Verordnung der Mittel und Maßnahmen vorliegt.

$34 \mathrm{Zu}$ dem Spektrum der von einem «allopathischen» Arzt verschriebenen und verwendeten Medikamente beispielsweise Balster 1990, 185-200. 
die zum Teil auch selbst hergestellt werden konnten. Pulver, Pillen und Tropfen wurden ebenso eingenommen. Für Seifen, Salben und Pflaster galt dasselbe. ${ }^{35}$

Auf eine medizinische Selbsthilfe weisen die diversen Nahrungsmittel hin, die man verwendete. Dazu gehörten neben Alkoholika, wie Schnaps, Likör, Brannt- und Rotwein, Tees und Wasser. ${ }^{36}$ Auch Essig, Öl, Schmalz und Butter, «Rinderpökel», «Roggenpapp», Hafergrütze, Hering, Speck, Pflaumen oder Feigen wurden als Arzneimittel gebraucht. ${ }^{37}$ Ebenso kamen Gewürze und Kräuter zum Einsatz. ${ }^{38}$

Andere Wirkstoffe waren Teil einer gängigen Selbstmedikation wie der Materia medica der praktizierenden Ärzte. Dies galt beispielsweise für «Leberthran», der üblicherweise zur Stärkung getrunken wurde, aber auch von Ärzten verordnet sein konnte. ${ }^{39}$ Viele Patienten hatten zuvor Chinin eingenommen oder Schwefel verwendet. ${ }^{40}$ Diese Mittel waren problemlos in Apotheken erhältlich, wurden aber ebenso im Rahmen einer ärztlichen Kur verschrieben. In ähnlicher Weise konnten einfache Bäder oder Waschungen selbständig oder unter professioneller Anleitung durchgeführt worden sein. ${ }^{41}$

35 In diverser Ratgeberliteratur wurden hierfür Rezepturen gegeben. Pillen wurden bei insgesamt 43 Patienten verwendet, Tropfen kamen in 46 Fällen zum Einsatz, darunter waren auch Stahltropfen oder die bekannten Hofmannstropfen. 28 Betroffene hatten Pulver eingenommen. Seifen waren von 19 und Pflaster bei 36 Kranken verwendet worden. Zur Herstellung von Pflastern Lutheritz 1825, 164-166, und Zeber 2001. Es hatten 286 Kranke zuvor eine Salbe verwendet. Berichtet wird von roten, gelben, grauen und weißen Salben.

36 Schnaps in 15 Fällen, Wein (Brannt- und Rotwein) bei 14 Kranken, Likör hatte ein Kranker getrunken, und ein weiterer sprach unbestimmt von «Spirituose». Zu Alkoholika als Hausmittel Lutheritz 1825,39-42, Mellin 1794,25-27. Wasser, auch in Form von Augenwasser oder mit den Zusätzen von Salz, Stahl, Blei, Arnika und Chlor oder in Form von Wasser aus diversen Kurbädern (besonders Carlsbad und Wildung), wurde bei 67 Patienten verwendet. Die Anwendungsgebiete von Wasser bei Mellin 1794,112-114. Tees waren von 31 Patienten getrunken worden. Dabei wurden beispielsweise auch Kamille und Fenchel verarbeitet. Lutheritz 1825, 28-37, Koch 1861, 16, Mellin 1794, 110.

37 Butter, Öl, Roggenpapp, Hafergrütze und Schmalz wurden als Vermischungsmaterial genutzt und bei Entzündungen aufgetragen. Hierzu Mellin 1794, 29 und 59.

38 Letztere wurden teilweise in Alkohol vermischt oder auch in Tees verwendet. Die Rede war beispielsweise von Wacholder, Anis, Wermut, Fenchel, Pfeffer, Salz oder Schöllkraut, Tausendgüldenkraut und Kamille.

39 Richter/Böhm 1989, 535.107 Kranke hatten angegeben, diesen eingenommen zu haben.

40 Chinin war bei Fiebererkrankungen eine häufig verwendete Medizin. Koch 1861, 117. Bei Friedrich von Bönninghausen konnte der Gebrauch bei wenigstens 105 Kranken (1,5\%) nachgewiesen werden. Äußerliche Schwefelanwendungen waren besonders bei Hauterkrankungen wie Krätze üblich. 110 Kranke (1,6\%) hatten damit Erfahrung gemacht.

41 Deren Zubereitung (auch mit Zusätzen wie Schwefel, Kleie, Salz oder Iod) und Indikationen wurde in diverser Ratgeberliteratur beschrieben. Zu den gebräuchlichsten medizinischen Bädern Medicinal-Kalender 1857, 109-112. 58 Betroffene hatten ein Bad durchgeführt. Die Waschungen, von denen 20 erwähnt werden, waren entweder mit reinem Wasser oder mit Zusätzen wie Kampfer, Anis, Schnaps oder Seife durchgeführt worden. IGM P 136 Fol. 239 verweist darauf, dass ein Arzt die Waschung des Kopfausschlages angeordnet hatte. 
Weitere Maßnahmen wie das Aufbringen von «Kataplasmen» oder «Fontanellen», das «Brennen», «Punktiren» oder «Abzapfen» sowie das Einführen eines Katheters weisen dagegen auf die Durchführung durch einen professionellen Mediziner hin. ${ }^{42}$ Einige Patienten trugen ein Bruchband oder einen Ring. ${ }^{43}$ Der Aderlass wurde nur noch von wenigen Kranken als eine vorherige Therapiemaßnahme erwähnt. ${ }^{44}$ Blutegel fanden aber nach wie vor Verwendung, und «geschröpft» wurde ebenfalls. So ersetzten diese «sanfteren» Blutentzugsmethoden die heftigeren Aderlässe. ${ }^{45}$

Diese Bandbreite von vorhergehenden Therapieversuchen durch Medikamente oder diverse Anwendungen wurde auch bei anderen Untersuchungen für das 19. Jahrhundert belegt. ${ }^{46}$ Von keinem von Bönninghausens Patienten wurden hingegen religiöse Heilversuche erwähnt. Auch die Vorschläge aus der «Dreckapotheke» der Volksmedizin beherzigte man kaum noch. Aber es mag sein, dass diese mehr und mehr in Verruf geratenen und zunehmend bekämpften Mittel in größerem Ausmaß verschwiegen wurden. ${ }^{47}$

Die Konsultation von «Kurpfuschern» oder Anwendungen «alternativer» Methoden wurden nur bei wenigen Kranken deutlich. ${ }^{48}$ Was vorangegangene homöopathische Kurversuche angeht, hatten immerhin 83 Patienten (1,2\%), die später zu Friedrich von Bönninghausen kamen, zuvor schon Erfahrungen mit der Lehre Hahnemanns gemacht. Oft ist aber nicht ersichtlich, welche Mittel gebraucht wurden oder wer die Behandlung durchführte.

Allerdings wird in den Krankengeschichten einiger Patienten deutlich, dass diese sich nicht an die Vorgabe hielten, neben der homöopathischen Kur

42 Kataplasmen wurden bei 39 Patienten verwendet. Fontanellen wurden neun Patienten gesetzt. Das «Brennen» erfolgte bei zehn Patienten, welche Wunden oder Geschwüre hatten. «Punktiren» und «Abzapfen» ist bei je einem Betroffenen genannt. Die vier «Katheterisierungen» waren alle bei Betroffenen vorgenommen worden, die an Blasenproblemen litten oder Schwierigkeiten beim Harnlassen hatten.

43 Ein solches kam bei Hernien oder Gebärmuttervorfällen zum Einsatz. Hierzu Baschin 2010, $122 \mathrm{f}$.

44 Dies sind 0,3\% der Betroffenen. Die Nennungen fallen mit einer Ausnahme alle in die Jahre bis 1875 .

4539 Kranke (0,6\%) hatten von den blutentziehenden Tieren Gebrauch gemacht. Näheres zu deren Verwendung bei Lutheritz 1825, 156f., Mellin 1794, 22-25. Bei 32 Patienten (0,5\%) war «Schröpfen» vermerkt.

46 Bei den Patienten Hahnemanns zum Beispiel Varady 1987, 338-341, Fischbach-Sabel 1998, 116. Für die Patienten des Vaters Baschin 2010, 87-101.

47 Allgemein zu magisch-religiösen Praktiken und dem Begriff der Volksmedizin Jütte 1996, 66-103. Zum Faktor des Verschweigens Ruisinger 2008, 126f.

48 Zur Diskussion des Begriffes «Alternativmedizin» und der Therapien, die gemeinhin darunter verstanden werden, ausführlich Jütte 1996. In lediglich 21 der 6832 Krankengeschichten klingt die Nutzung von nicht approbierten Heilpersonen beziehungsweise nicht gebilligten Mitteln an. Vier weitere Kranke hatten Erfahrungen mit der Anwendung von Elektrizität gemacht, ein anderer Patient mit dem Mesmerismus. 
gänzlich auf «allopathische» Anwendungen zu verzichten. Andere Betroffene hatten nach einer erfolgreichen homöopathischen Kur zunächst wieder zu den üblichen Mitteln gegriffen, ehe sie es später noch einmal bei Friedrich von Bönninghausen mit der Lehre Hahnemanns probierten. ${ }^{49}$

Selbstverständlich waren auch die für den Heilberuf ausgebildeten approbierten Ärzte und Chirurgen um Rat gefragt worden. ${ }^{50}$ Mehr als 150 Patienten hatten sich einem im weitesten Sinne chirurgischen Eingriff unterzogen, bevor sie zu Bönninghausen kamen..$^{51}$ Doch handelte es sich kaum um schwerwiegende Operationen im Körperinneren, sondern meist um das oberflächliche Eröffnen von Geschwulsten oder Gewächsen, beispielsweise im Hals- und Nackenbereich oder an den Fingern. Die meisten Kranken berichteten, dass ihnen Zähne gezogen worden waren. ${ }^{52}$ Die Entfernung von Polypen in Nase und Ohren, wie auch der Rachenmandeln, gehörte zu den neueren chirurgischen Eingriffsbereichen. ${ }^{53}$ Gleiches gilt für die Augenheilkunde. ${ }^{54}$ Manchmal sollte der Homöopath Nachwirkungen der Operation behandeln. Andererseits kamen auch Betroffene, die hofften, durch die homöopathischen Mittel im letzten Moment einen Eingriff oder gar eine Amputation zu verhindern. ${ }^{55}$

Mehr als 500 Kranke hatten zuvor Rat bei der «schulmedizinischen» Konkurrenz des Homöopathen geholt. Gelegentlich waren dabei mehrere Ärzte konsultiert worden. ${ }^{56}$ Wenn die Betroffenen nicht unmittelbar in Münster wohnten, waren zunächst die Mediziner «vor Ort» um Rat gefragt worden. Doch konnten die befragten Ärzte wohl nicht zur Zufriedenheit der Patienten handeln, denn diese wandten sich, wie in den Journalen ersichtlich,

49 Bei wenigstens 28 der 83 Patienten waren neben den zuvor verwendeten homöopathischen Mitteln auch andere «allopathische» Anwendungen vermerkt worden.

50 Hebammen zählten ebenfalls zu den approbierten Medizinalpersonen. Deren Konsultation wurde jedoch nur bei zwei Patienten deutlich.

51 In 174 Krankengeschichten (2,5\%) wird ein chirurgischer Eingriff erwähnt.

5241 Patienten hatten sich einen oder mehrere Zähne ziehen lassen. Am Mund- und Kieferbereich wurden drei Eingriffe durchgeführt, und bei drei Betroffenen war am Gaumen operiert worden. Zwei Kranke hatten sich im Gesicht operieren lassen, und bei zwei weiteren waren Geschwulste an der Zunge chirurgisch behandelt worden.

53 Eckart 1990, 237. Eingriffe an Nase und Ohren waren bei vier beziehungsweise acht Betroffenen durchgeführt worden. Die Mandeln hatte man bei zwei Patienten «exstirp.(iert)».

54 Zur Entwicklung der Augenheilkunde Münchow 1984. Auch in Münster gab es eine derartige Spezialklinik, hierzu Schwanitz 1990, 96. Insgesamt berichteten zwölf Patienten von Operationen am Auge.

55 Es wurde von sechs geplanten Eingriffen berichtet. In zwei Fällen waren bereits Amputationen durchgeführt worden, und Bönninghausen behandelte Nachwirkungen.

56 Bei 537 Patienten wurde in der Erstanamnese oder in der Zeile «Allop.(athisch) gebr.(aucht)» auf eine Behandlung durch einen oder mehrere Ärzte verwiesen. Oft wurde der Name nicht genannt. Bei wenigstens 41 der Betroffenen war eine Kur von mehreren unbekannten Ärzten durchgeführt worden. Dabei waren es meist zwischen zwei und drei zuvor konsultierte Ärzte, aber deren Anzahl konnte auch bis zu 20 reichen. 
an Bönninghausen. Auch zahlreiche Ärzte aus Münster waren konsultiert worden, bevor man den Homöopathen ins Vertrauen zog. Deutlich wird, dass die Kranken, gerade bei länger anhaltenden Beschwerden, durchaus bereit waren, einen beziehungsweise mehrere Ärzte aufzusuchen. Erst dann wandten sich die Betroffenen an Bönninghausen.

Krankenhäuser und Kurbäder waren als Institutionen des Medizinalsystems genutzt worden. Insbesondere das Aufsuchen diverser Kurorte war bis zum 1. Weltkrieg in Mode. ${ }^{57}$ Entsprechend muss es nicht wundern, dass viele von Bönninghausens späteren Patienten dieses Angebot genutzt hatten. Allerdings war ein solcher Aufenthalt eher Wohlhabenderen vorbehalten. ${ }^{58}$ Krankenhäuser hatten zum Ausgang des 19. Jahrhunderts teilweise den Charakter als Armen- und Pflegeanstalten verloren. Dennoch begaben sich eher Kranke dorthin, die zu Hause nicht von Angehörigen gepflegt werden konnten. ${ }^{59}$ Was den Aufenthalt in einem Krankenhaus angeht, mag es erstaunen, dass der Anteil derjenigen, die zuvor in einer solchen Institution gewesen waren, recht gering ist. Doch führt auch heute nur ein Bruchteil der Erkrankungen zu einer Krankenhausbehandlung. ${ }^{60}$

\section{Motive und Verhalten der Patienten auf dem medizinischen Markt}

Da Friedrich von Bönninghausen nachweislich nicht der einzige Arzt in Münster war, sondern sich einer sehr großen Konkurrenz ausgesetzt sah, bleibt zu fragen, was man aus den vorliegenden Krankengeschichten über die Motive der Einzelnen, sich an diesen Homöopathen zu wenden, erfahren kann. Zweifelsohne war bekannt, dass Bönninghausen nach der Lehre Hahnemanns therapierte, so dass man bei den Betroffenen eine «bewusste» Entscheidung für das «alternative» Konzept voraussetzen kann. ${ }^{61}$ Welche Überlegungen genau zu der zumindest einmaligen Konsultation des Homöopathen geführt haben, ist aber im Einzelfall nicht bekannt. Doch lassen sich aus den verschiedenen Aufzeichnungen einige Motive erschließen.

57 Nicht nur die Anzahl der Gäste in den einzelnen Kurorten nahm zu, auch die Anzahl der Kurorte selbst stieg. Dies ist beispielsweise dem Bericht über das Sanitätswesen des Preußischen Staates 1902 zu entnehmen. Ein Kurbad hatten 72 Kranke zuvor besucht.

58 Bei den Betroffenen, die angegeben hatten, ein Kurbad besucht zu haben, war in 32 Fällen eine Schichtzuordnung möglich. Davon wurden 18 der Mittel- und zehn der Oberschicht zugewiesen. Vier Patienten waren Angehörige der Unterschicht.

59 Zur Entwicklung des Krankenhauswesens Labisch/Spree 1996, Eckart 1990, 206-212 sowie 242-245.

6032 Patienten von Bönninghausen waren zuvor in einem Krankenhaus gewesen. Das entspricht einem Anteil von 0,5\% der Patienten. Als bildliche Darstellung zu diesen Verhältnissen Larsen 1991, 142.

61 Ähnliche Überlegungen Stahl 1995, 216. 
Letztendlich hatte die Mehrheit der Kranken zuvor Erfahrungen mit «allopathischen» Therapieversuchen gemacht, sei es in Eigenregie oder unter der Anleitung eines Arztes. Bei Betroffenen, die aus der Umgebung Münsters kamen, waren erst die Ärzte vor Ort konsultiert worden, bevor der Weg in die Provinzialhauptstadt angetreten wurde. Insofern waren die wenigsten, als sie ihre Wahl für Bönninghausen fällten, überzeugte Anhänger der Homöopathie. Am ehesten trifft dies auf den kleinen Bruchteil derjenigen zu, die schon zuvor entsprechende Mittel ausprobiert oder andere Homöopathen um Rat gefragt hatten. Doch zeigt sich bei deren Geschichten zum Teil, dass sie nicht bedingungslose Anhänger der Lehre Hahnemanns waren.

Plausibel ist die Annahme, dass viele der Betroffenen, die bei Bönninghausen in der Praxis erschienen, bisher nicht mit den gewählten Mitteln zufrieden waren. Deutlich wird das an denjenigen, die angaben, die seitherigen Therapieversuche hätten zu einer steten Verschlimmerung geführt oder seien ohne Erfolg geblieben. ${ }^{62} \mathrm{Ob}$ dann die Homöopathie als letzter Ausweg gesehen wurde oder ob weitere Gründe für deren Gebrauch sprachen, kann nicht mehr entschieden werden. Häufig wird man von Ersterem ausgehen müssen. ${ }^{63}$

Misslungene Operationen oder der Tod von Angehörigen im Zusammenhang mit einer offenkundig erfolglosen «allopathischen» Therapie waren starke Motive, sich nach einer anderen Heilmethode umzusehen. Wenn beispielsweise ein durchgeführter chirurgischer Eingriff zum Verlust eines Auges geführt hatte, kann man sich vorstellen, weshalb der Betroffene zunächst alle anderen Möglichkeiten ausschöpfte, ehe er sich erneut zu einer Operation durchrang. In anderen Fällen hatten Eltern bereits ein Kind an Diphtherie oder Keuchhusten trotz ärztlicher Hilfe verloren und waren nun auf der Suche nach einer weiteren Behandlungsweise für den an denselben Symptomen leidenden Nachwuchs. ${ }^{64}$

62 Die explizite Angabe mit «Verschl.(immerung)» findet sich in drei Krankengeschichten. Die Abkürzung «o.(hne) E.(rfolg)» gibt es in sechs Behandlungen, sinngemäß trifft dies auch auf weitere Behandlungen zu.

63 Darüber machten sich auch die Homöopathen selbst keine Illusionen. Baschin 2010, 143. Sehr deutlich beispielsweise die dahingehende Aussage in IGM P 123 Fol. 30: "Allerlei; zuletzt homöop.(athisch)». Dies gilt sicherlich dann, wenn die Krankheiten als «chronisch» einzustufen sind. Explizit wurde bei 26 Patienten von «chronischen» Leiden gesprochen. Bei 1561 der 6832 Kranken (22,8\%) dauerte das Leiden schon seit mindestens einem Jahr. Dies würde ebenfalls für eine Einstufung des Leidens als «chronisch» sprechen. Das trifft heute in noch viel größerem Maße zu. Hierzu Sharma 1995, 24-26.

64 Bei wenigstens 18 Krankengeschichten wird der Tod eines Angehörigen an den ähnlichen Symptomen, wie sie Bönninghausen nun behandeln sollte, erwähnt. Das Beispiel der misslungenen Augenoperation in IGM P 148 Fol. 138, auch IGM P 118 Fol. 475. 
Auch wenn die bisher konsultierten Ärzte die Kranken aufgegeben oder deren Leiden für unheilbar erklärt hatten, liegt die Wendung zur Lehre Hahnemanns als letztem Strohhalm nahe. ${ }^{65}$ Ähnliches mag gelten, wenn es um die Absicht ging, eine Operation oder gar Amputation zu verhindern. In einem solchen Fall konnte ein Ausprobieren der Globuli im Idealfall den Eingriff abwenden, schlimmer konnte die Situation aber kaum werden. ${ }^{66}$

Was sich hingegen nicht in den Notizen niederschlägt, ist eine Konsultation aus finanziellen Erwägungen. Gemeinhin galt die Homöopathie als eine relativ günstige Behandlungsmethode. ${ }^{67}$ Es ist aber weder bekannt, dass Bönninghausen kostenlos behandelte, noch waren seine Tarife vermutlich wesentlich günstiger als diejenigen seiner «schulmedizinischen» Konkurrenz. ${ }^{68}$ Es kann nicht genau bestimmt werden, wie teuer eine Konsultation bei Bönninghausen war. Allem Anschein nach staffelte der Arzt die Kosten einer Behandlung entsprechend den finanziellen Möglichkeiten seiner Patienten und hielt sich an die durch die preußische Medizinaltaxe vorgegebenen Rahmenbeträge. Da Bönninghausen nicht als Kassenarzt praktizierte, war eine Übernahme der Behandlungskosten durch diese nicht möglich. Daher fehlen für dieses ökonomische Argument die Belege, auch wenn es nicht ganz ausgeschlossen werden kann. ${ }^{69}$

Insofern waren die meisten Kranken kaum «überzeugte» Nutzer der Homöopathie. ${ }^{70}$ Berücksichtigt man, dass zudem fast $40 \%$ aller Behandlungen bereits nach einer Konsultation wieder beendet waren, kann man feststellen, dass es sich in den meisten Fällen um eine in höchstem Maße sporadische Nutzung der Methode Hahnemanns handelte. ${ }^{71}$ Gerade bei der dokumentierten einmaligen beziehungsweise der kurzzeitigen Behandlung mag der Gang zum Homöopathen eher als eine zusätzliche Option genutzt

65 In sechs Krankengeschichten wird Ähnliches deutlich.

66 In insgesamt sieben Krankengeschichten klingt ein solches Motiv an.

67 Beispielsweise Stahl 1995, 216, Jütte 1996, 204 und 220, sowie Dinges 2002, 8f.

68 Beispielsweise behandelten Samuel Hahnemann und Clemens von Bönninghausen stets Patienten kostenlos. Vergleiche Jütte 1996, 218, und Baschin 2010,368f., ebenso der Verweis bei Stahl 1995, 216. Über die Honorarforderung der übrigen Ärzte in Münster in dieser Zeit ist nichts bekannt.

69 Für lediglich 88 Kranke ist ein Vermerk über Honorarleistungen in den Krankengeschichten verzeichnet. Auch ein vorhandenes «Conto-Buch» gibt nur über einen Bruchteil der Behandlungen Auskunft. Differenzierte Auswertungen stehen noch aus. Die preußische Medizinaltaxe findet sich beispielsweise in Medicinal-Kalender 1857, 10-14. Daher folgte Friedrich von Bönninghausen auch in diesem Gesichtspunkt der Praxis seines Vaters. Vergleiche Baschin 2010,368-382.

70 Die im Folgenden genannten «Typen» von Patienten beziehungsweise deren Nutzungsverhalten lehnen sich an Dinges 2002,18-20, an.

71 Von den 6832 Patienten hatten 2622 lediglich eine Konsultation. Dies trifft jedoch auch auf «allopathische» Arztpraxen zu. Baschin 2010, 336f. mit weiterer Literatur. 
worden sein, ohne dass man sich völlig darauf einlassen wollte. ${ }^{72}$ Freilich gab es auch diejenigen, die über Jahre hinweg immer wieder kamen, wobei hier selten geklärt werden kann, inwieweit diese in Erkrankungsfällen ausschließlich Bönninghausen aufsuchten oder doch weiterhin die «Schulmedizin» nutzten. Daher wäre es in diesen Fällen zu unsicher, von zur Homöopathie «konvertierten» Kranken auszugehen. So kann man die Inanspruchnahme Bönninghausens in den meisten Fällen darauf zurückführen, dass neben den vielen anderen Möglichkeiten auch die Homöopathie ausprobiert wurde. Half sie nicht, wandte man sich den anderen Angeboten zu. Infolgedessen kann man die Therapieweise kaum als eine wirkliche «Alternative» auf dem medizinischen Markt Münsters sehen. Vielmehr war sie ein «komplementär» genutztes Konzept.

\section{Archivsiglen}

IGM Institut für Geschichte der Medizin der Robert Bosch Stiftung Stuttgart

StAM Staatsarchiv Münster, Landesarchiv NRW Abteilung Westfalen

StdAM Stadtarchiv Münster

\section{Bibliographie}

Adreß-Buch der Stadt Münster für 1889, Band 2 (Münster 1889).

Adreß-Buch der Stadt Münster in Westf.(alen) für 1905, Band 2 (Münster 1905)

Balster, Wolfgang, Medizinische Wissenschaft und ärztliche Praxis im Leben des Bochumer Arztes Karl Arnold Kortum (1745-1824) (Bochum 1990) (Med. Diss.)

Baschin, Marion, Wer lässt sich von einem Homöopathen behandeln? Die Patienten des Clemens Maria Franz von Bönninghausen (1785-1864), Medizin, Gesellschaft und Geschichte, Beiheft 37 (Stuttgart 2010)

Dinges, Martin, «Arztpraxen 1500-1900. Zum Stand der Forschung», in: Elisabeth DietrichDaum/Martin Dinges/Robert Jütte/Christine Roilo (Hrsg.), Arztpraxen im Vergleich. 18.-20. Jahrhundert, Veröffentlichungen des Südtiroler Landesarchivs 26 (Innsbruck/Wien/ Bozen 2008) 23-62

Dinges, Martin, "Introduction. Patients in the History of Homoeopathy", in: Martin Dinges (Hrsg.), Patients in the History of Homoeopathy, European Association for the History of Medicine and Health Network Series (Sheffield 2002) 1-32.

Eckart, Wolfgang, Geschichte der Medizin (Berlin/Heidelberg/New York 1990)

Eulenberg, Hermann, Das Medicinalwesen in Preussen, Nach amtlichen Quellen (Berlin ${ }^{3} 1874$ )

Fischbach-Sabel, Ute, Samuel Hahnemann. Krankenjournal D34 (1830), Kommentarband zur Transkription (Heidelberg 1998)

Graf, Eduard, Das ärztliche Vereinswesen in Deutschland und der Deutsche Ärztevereinsbund, Festschrift dem 10. Internationalen Medizinischen Kongress gewidmet (Leipzig 1890)

72 Ähnliches stellte Stahl 1995, 216 für die Patienten des Homöopathen Gauwerky fest. Es trifft ebenso auf die Kranken zu, die den Vater Clemens von Bönninghausen konsultiert hatten. Baschin 2010,141-145. 
Hahnemann, Samuel, Organon der Heilkunst, <Aude sapere >, in der Standardausgabe der 6. Auflage. Herausgegeben von Josef Schmidt (Stuttgart 1999)

Huerkamp, Claudia, Der Aufstieg der Ärzte im 19. Jahrhundert, Vom gelehrten Stand zum professionellen Experten, Das Beispiel Preußens, Kritische Studien zur Geschichtswissenschaft 68 (Göttingen 1985)

Jütte, Robert, Geschichte der Alternativen Medizin, Von der Volksmedizin zu den unkonventionellen Therapien von heute (München 1996)

Jütte, Robert (Hrsg.), Geschichte der deutschen Ärzteschaft, Organisierte Berufs- und Gesundheitspolitik im 19. und 20. Jahrhundert (Köln 1997)

Jungnitz, Bernhard, Die konfessionellen Krankenhäuser der Stadt Münster im achtzehnten und neunzehnten Jahrhundert, Studien zur Geschichte des Krankenhauswesens 18 (Herzogenrath 1981)

Koch, August, Die bewährtesten Hausmittel der Deutschen, Aus den Papieren eines Militärarztes (Leipzig ${ }^{3} 1861$ )

Krabbe, Wolfgang, «Wirtschafts- und Sozialstruktur einer Verwaltungsstadt des 19. Jahrhunderts. Das Beispiel der Provinzialhauptstadt Münster», in: Kurt Düwell/Wolfgang Köllmann (Hrsg.), Rheinland-Westfalen im Industriezeitalter, Band 1, Von der Entstehung der Provinzen bis zur Reichsgründung (Wuppertal 1983) 197-206

Labisch, Alfons/Reinhard Spree (Hrsg.), Einem jeden Kranken in einem Hospitale sein eigenes Bett, Zur Sozialgeschichte des Allgemeinen Krankenhauses in Deutschland im 19. Jahrhundert (Frankfurt a.M./New York 1996)

Lachmund, Jens/Gunnar Stollberg, Patientenwelten, Krankheit und Medizin vom späten 18. bis zum frühen 20. Jahrhundert im Spiegel von Autobiographien (Opladen 1995)

Larsen, Øivind, "Case Histories in Nineteenth-Century Hospitals, What Do They Tell the Historians? Some Methodological Considerations with Special Reference to McKeown's Criticism of Medicine", in: Medizin, Gesellschaft und Geschichte 10 (1991) 127-148

Loetz, Francisca, Vom Kranken zum Patienten, ‘Medikalisierung> und medizinische Vergesellschaftung am Beispiel Badens 1750-1850, in: Medizin, Gesellschaft und Geschichte Beiheft 2 (Stuttgart 1993)

Lutheritz, Karl, Hausapotheke oder medicinisches Noth- und Hülfsbüchlein für Nichtärzte zur Kenntniß, Wahl und Anwendungsart der wichtigsten, und durch sichere Erfahrung bei innerlichen und äußerlichen Krankheiten bewährt gefundenen Hausmittel (Meißen 1825)

Medicinal-Kalender für den Preussischen Staat auf das Jahr 1857 (Berlin 1857)

Mellin, Christoph, Die Hausmittel, Eine Sammlung der besten, gemeinnützigsten und sichersten Mittel, die Gesundheit des Menschen zu erhalten, und den Krankheiten gehörig vorzubeugen (Grätz 1794)

Münchow, Wolfgang, Geschichte der Augenheilkunde (Stuttgart ${ }^{2} 1984$ )

Richter, Hans/Manfred Böhm (Hrsg.), Pharmazeutisch-medizinisches Lexikon, Zwei Bände (Berlin 1989)

Ruisinger, Marion, Patientenwege, Die Konsiliarkorrespondenz Lorenz Heisters (1683-1758) in der Trew-Sammlung Erlangen, Medizin, Gesellschaft und Geschichte Beiheft 28 (Stuttgart 2008)

Sanders, Bernhard, «Beitrag zur Geschichte der Homöopathie im Land Westfalen», Allgemeine Homöopathische Zeitung 209 (1964) 334-341

Sanitätswesen des Preussischen Staates während der Jahre 1895, 1896 und 1897. Bearbeitet von der Medizinal-Abteilung des Ministeriums (Berlin 1902)

Schlich, Thomas/Reinhart Schüppel, «Gibt es einen Aufschwung für die Homöopathie. Von der Schwierigkeit, die Verbreitung der Homöopathie unter Ärzten festzustellen», in: Martin Dinges (Hrsg.), Homöopathie. Patienten, Heilkundige, Institutionen, Von den Anfängen bis heute (Heidelberg 1996) 210-227

Schwanitz, Hedwig, Krankheit, Armut, Alter, Gesundheitsfürsorge und Medizinalwesen in Münster während des 19. Jahrhunderts. Quellen und Forschungen zur Geschichte der Stadt Münster Neue Folge 14 (Münster 1990)

Sharma, Ursula, Complementary Medicine Today, Practitioners and Patients (London/New York 21995)

Spree, Reinhard, «Quantitative Aspekte der Entwicklung des Krankenhauswesens im 19. und 20. Jahrhundert», in: Alfons Labisch/Reinhard Spree (Hrsg.), Einem jeden Kranken in einem 
Hospitale sein eigenes Bett, Zur Sozialgeschichte des Allgemeinen Krankenhauses in Deutschland im 19. Jahrhundert (Frankfurt a.M./New York 1996) 51-88

Spree, Reinhard, «Kurpfuscherei. Bekämpfung und ihre sozialen Funktionen während des 19. und 20. Jahrhunderts», in: Alfons Labisch/Reinhard Spree (Hrsg.), Medizinische Deutungsmacht im sozialen Wandel (Bonn 1989) 103-121

Stahl, Martin, «Zur Geschichte der <Vereinigung homöopathischer Aerzte Rheinlands und Westphalens»», Medizin, Gesellschaft und Geschichte 14 (1995) 195-218

Teuteberg, Hans-Jürgen, Materialien zur Bevölkerungsgeschichte Münsters 1816-1945, Beiträge zur Statistik Münsters 59 (Münster 1993a)

Teuteberg, Hans-Jürgen, «Bevölkerungsentwicklung und Eingemeindungen (1816-1945)», in: Franz-Josef Jakobi (Hrsg.), Geschichte der Stadt Münster, Band 2 (Münster 1993b) 331-386

Varady, Helene, Die Pharmakotherapie Samuel Hahnemanns in der Frühzeit der Homöopathie. Edition und Kommentar des Krankenjournals Nr. 5 (1803-1806), Zwei Bände (München 1987) (Med. Diss.)

Walter, Bernd, «Von der fürstbischöflichen Haupt- und Residenzstadt zur preußischen Provinzialhauptstadt (1815-1835)» in: Franz-Josef Jakobi (Hrsg.), Geschichte der Stadt Münster, Band 2 (Münster 1993) 47-78

Zeber, Ulrike, Die Geschichte des Pflasters, Von der traditionellen Arzneiform Pflaster zum Heftpflaster, in:Heidelberger Schriften zur Pharmazie- und Naturwissenschaftsgeschichte 18 (Stuttgart 2001)

140 Gesnerus $69(2012)$ 